\title{
Article
}

\section{Moments of generalized order statistics for Pareto-Rayleigh distribution}

\author{
M. Alam ${ }^{1}$, R. U. Khan ${ }^{1}$ and Z. Vidović ${ }^{2, *}$ \\ 1 Department of Statistics and Operations Research, Aligarh Muslim University, Aligarh-202 002, India. \\ 2 Teacher Education Faculty, Belgrade 11000, Serbia. \\ * Correspondence: zoran.vidovic@uf.bg.ac.rs
}

Academic Editor: Christophe Chesneau

Received: 20 July 2021; Accepted: 12 September 2021; Published: 9 October 2021.

Abstract: In this paper, we derive the explicit expressions for single and product moments of generalized order statistics from Pareto-Rayleigh distribution using hypergeometric functions. Also, some interesting remarks are presented.

Keywords: Generalized order statistics; Pareto-Rayleigh distribution; Single moments; Product moments; Hypergeometric functions.

MSC: 62G30; 33C90.

\section{Introduction}

amps [1] introduced the concept of generalized order statistics (gos) as follows: Let us note $n \in N$, $k \geq 1$, and $\tilde{m}=\left(m_{1}, m_{2}, \ldots, m_{n-1}\right) \in \mathfrak{R}^{n-1}, 1 \leq r \leq n-1$, such that

$$
\gamma_{r}=k+n-r+\sum_{j=r}^{n-1} m_{j}>0 \text { for } 1 \leq r \leq n-1 .
$$

The random variables $X(1, n, \tilde{m}, k), X(2, n, \tilde{m}, k), \ldots, X(n, n, \tilde{m}, k)$ are said to be $g o s$ from a continuous population with cumulative distribution function $(c d f) F(x)$ and probability distribution function $(p d f) f(x)$ if their joint $p d f$ is of the form

$$
k\left(\prod_{j=1}^{n-1} \gamma_{j}\right)\left(\prod_{i=1}^{n-1}\left[\bar{F}\left(x_{i}\right)\right]^{m_{i}} f\left(x_{i}\right)\right)\left[\bar{F}\left(x_{n}\right)\right]^{k-1} f\left(x_{n}\right),
$$

defined on the cone $F^{-1}(0+)<x_{1} \leq x_{2} \leq \ldots \leq x_{n}<F^{-1}(1)$ of $\mathfrak{R}^{n}$, where $\bar{F}(x)=1-F(x)$.

The model of gos contains special cases such as ordinary order statistics $\left(\gamma_{i}=n-i+1 ; i=\right.$ $1,2, \ldots, n$ i.e. $\left.m_{1}=m_{2}=\cdots=m_{n-1}=0, k=1\right), k$-th record values $\left(\gamma_{i}=k\right.$ i.e., $m_{1}=m_{2}=\cdots=$ $\left.m_{n-1}=-1, k \in N\right)$, sequential order statistics $\left(\gamma_{i}=(n-i+1) \alpha_{i} ; \alpha_{1}, \alpha_{2}, \ldots, \alpha_{n}>0\right)$, order statistics with non-integer sample size $\left(\gamma_{i}=\alpha-i+1 ; \alpha>0\right)$, Pfeifer's record values $\left(\gamma_{i}=\beta_{i} ; \beta_{1}, \beta_{2}, \ldots, \beta_{n}>0\right)$ and progressive type II censored order statistics $\left(\gamma_{r}=n-r+1+\sum_{i=r}^{l} m_{i}, 1 \leq r \leq l \leq n, m_{i} \in N, k=m_{n}+1\right)$, see [1-3].

Here we shall obtain the results for $\gamma_{i} \neq \gamma_{j}$ and then deduce the results for $\gamma_{i}=\gamma_{j}\left(m_{1}=m_{2}=\cdots=\right.$ $\left.m_{n-1}=m \neq-1\right)$.

Therefore, we will consider two cases:

Case I: $\gamma_{i}=\gamma_{j}\left(m_{1}=m_{2}=\cdots=m_{n-1}=m \neq-1\right)$ [1].

Case II: $\gamma_{i} \neq \gamma_{j}, i \neq j i, j=1,2, \ldots, n-1$ [2].

Case I: The $p d f$ of $r$-th gos $X(r, n, m, k)$, is given by

$$
f_{X(r, n, m, k)}(x)=\frac{C_{r-1}}{(r-1) !}[\bar{F}(x)]^{\gamma_{r}-1} f(x) g_{m}^{r-1}(F(x)),
$$


and the joint $p d f$ of $X(r, n, m, k)$ and $X(s, n, m, k), 1 \leq r<s \leq n$, is given by

$$
\begin{aligned}
f_{X(r, n, m, k), X(s, n, m, k)}(x, y)= & \frac{C_{s-1}}{(r-1) !(s-r-1) !}[\bar{F}(x)]^{m} g_{m}^{r-1}(F(x)) \\
& \times\left[h_{m}(F(y))-h_{m}(F(x))\right]^{s-r-1}[\bar{F}(y)]^{\gamma_{s}-1} f(x) f(y), \quad x<y,
\end{aligned}
$$

where

$$
\begin{aligned}
& C_{r-1}=\prod_{i=1}^{r} \gamma_{i}, \quad \gamma_{i}=k+(n-i)(m+1), \\
& h_{m}(x)= \begin{cases}-\frac{1}{m+1}(1-x)^{m+1} & , \quad m \neq-1 \\
-\ln (1-x) & , m=-1\end{cases}
\end{aligned}
$$

and

$$
g_{m}(x)=h_{m}(x)-h_{m}(0)=\int_{0}^{x}(1-t)^{m} d t, x \in[0,1) .
$$

Case II: The $p d f$ of $r-$ th $g o s X(r, n, \tilde{m}, k)$, is given by

$$
f_{X(r, n, \tilde{m}, k)}(x)=C_{r-1} \sum_{i=1}^{r} a_{i}(r)[\bar{F}(x)]^{\gamma_{i}-1} f(x)
$$

with the joint $p d f$ of $X(r, n, \tilde{m}, k)$ and $X(s, n, \tilde{m}, k), 1 \leq r<s \leq n$,

$$
f_{X(r, n, \tilde{m}, k), X(s, n, \tilde{m}, k)}(x, y)=C_{s-1}\left[\sum_{i=r+1}^{s} a_{i}^{(r)}(s)\left\{\frac{\bar{F}(y)}{\bar{F}(x)}\right\}^{\gamma_{i}}\right]\left[\sum_{i=1}^{r} a_{i}(r)\{\bar{F}(x)\}^{\gamma_{i}}\right] \frac{f(x)}{\overline{\bar{F}}(x)} \frac{f(y)}{\bar{F}(y)}
$$

where

$$
\begin{aligned}
& C_{r-1}=\prod_{i=1}^{r} \gamma_{i}, \\
& \gamma_{r}=k+n-r+\sum_{j=r}^{n-1} m_{j}, \\
& a_{i}(r)=\prod_{j=1}^{r} \frac{1}{\left(\gamma_{j}-\gamma_{i}\right)}, \quad j \neq i, \quad \gamma_{j} \neq \gamma_{i}, \quad 1 \leq i \leq r \leq n, \\
& a_{i}^{(r)}(s)=\prod_{j=r+1}^{n} \frac{1}{\left(\gamma_{j}-\gamma_{i}\right)}, \quad j \neq i, \quad \gamma_{j} \neq \gamma_{i}, \quad r+1 \leq i \leq s \leq n .
\end{aligned}
$$

For $m_{1}=m_{2}=\cdots=m_{n-1}=m \neq-1$, it can be shown that [3]:

$$
a_{i}(r)=\frac{(-1)^{r-i}}{(m+1)^{r-1}(r-1) !}\left(\begin{array}{c}
r-1 \\
r-i
\end{array}\right)
$$

and

$$
a_{i}^{(r)}(s)=\frac{(-1)^{s-i}}{(m+1)^{s-r-1}(s-r-1) !}\left(\begin{array}{c}
s-r-1 \\
s-i
\end{array}\right) .
$$

In this paper we are interested in a situation when a random variable $X$ follows the Pareto-Rayleigh(P-R) distribution with $p d f$

$$
f(x ; \alpha, \sigma)=\frac{\alpha}{\sigma^{2}} x\left(1+\frac{x^{2}}{2 \sigma^{2}}\right)^{-(\alpha+1)} \quad x>0, \alpha>1, \text { and } \sigma>0,
$$

and with $d f$

$$
F(x ; \alpha, \sigma)=1-\left(1+\frac{x^{2}}{2 \sigma^{2}}\right)^{-\alpha} \quad x>0, \alpha>1, \text { and } \sigma>0 .
$$

In view of (8) and (9),

$$
\left(1+\frac{x^{2}}{2 \sigma^{2}}\right) f(x)=\frac{\alpha}{\sigma^{2}} x \bar{F}(x)
$$


Pareto-Rayleigh distribution can be seen as a member of Transformed-Transformer family (or T-X family) of distributions proposed by Alzaatreh et al., [4]. This distribution is recognized as a good model for fitting various lifetime data, see Jebeli and Deiri [5]. This is also confirmed in [6] were a comparative study on the performance of Pareto-Rayleigh distribution against biased Lomax distribution was conducted. Further, for more details on Pareto-Rayleigh distribution one can see [7-9].

Exact moments expressions of gos for different distributions have been obtained by literature. Some examples are exponentiated Log-logistic distribution, Burr type XII distribution, linear exponential distribution, Erlang-truncated exponential distribution, Burr distribution, power function distribution, type II exponentiated Log-logistic distribution, extended exponential distribution, generalized Pareto distribution, q-Weibull distribution; see, respectively, Athar and Nayabuddin [10], Khan et al., [11], Ahmad [12], Khan et al., [13], Khan and Khan [3], Kumar and Khan [14], Kumar [15], Kumar and Dey [16], Malik and Kumar [17], Singh et al., [18] and Kumar et al., [19].

In this paper, we have derived explicit expression for single and product moments of Pareto-Rayleigh distribution based on gos.

\section{Relations for Product Moments}

In this section, we derive the exact expressions for product moments of generalized order statistics in the following theorems. Before coming to the main result, the following lemma is proved.

Lemma 1. For the Pareto-Rayleigh distribution with $c d f(1.9)$ next relations holds

$$
\Phi_{j, l}(a, b)=\frac{\left(2 \sigma^{2}\right)^{\left(\frac{j+l}{2}+2\right)}}{2(j+2)} B\left(\frac{j+l}{2}+2, \alpha b-\frac{l}{2}\right)_{3} F_{2}\left(\frac{j}{2}+1,1-a \alpha+\frac{j}{2}, \frac{j+l}{2}+2 ; \frac{j}{2}+2, \frac{j}{2}+\alpha b+2 ; 1\right)
$$

where

$$
\Phi_{j, l}(a, b)=\int_{0}^{\infty} \int_{0}^{y} \frac{x^{j+1}}{\left(1+\frac{x^{2}}{2 \sigma^{2}}\right)^{a \alpha+1}} \frac{y^{l+1}}{\left(1+\frac{y^{2}}{2 \sigma^{2}}\right)^{\alpha b+1}} d x d y
$$

and

$$
{ }_{p} F_{q}\left[a_{1}, \ldots, a_{p} ; b_{1}, \ldots, b_{q} ; x\right]=\sum_{r=0}^{\infty}\left[\prod_{j=1}^{p} \frac{\Gamma\left(a_{j}+r\right)}{\Gamma\left(a_{j}\right)}\right]\left[\prod_{j=1}^{q} \frac{\Gamma\left(b_{j}\right)}{\Gamma\left(b_{j}+r\right)}\right] \frac{x^{r}}{r !},
$$

for $p=q+1$ and $\sum_{j=1}^{q} b_{j}-\sum_{j=1}^{p} a_{j}>0$.

Proof. We have

$$
\Phi_{j, l}(a, b)=\int_{0}^{\infty} \frac{y^{l+1}}{\left(1+\frac{y^{2}}{2 \sigma^{2}}\right)^{\alpha b+1}}\left[\int_{0}^{y} \frac{x^{j+1}}{\left(1+\frac{x^{2}}{2 \sigma^{2}}\right)^{a \alpha+1}} d x\right] d y
$$

Let

$$
B(y)=\int_{0}^{y} \frac{x^{j+1}}{\left(1+\frac{x^{2}}{2 \sigma^{2}}\right)^{a \alpha+1}} d x
$$

Substituting $1-u=\frac{1}{\left(1+\frac{x^{2}}{2 \sigma^{2}}\right)}$ in (14), we get

$$
\begin{aligned}
B(y) & =\frac{\left(2 \sigma^{2}\right)^{\left(1+\frac{j}{2}\right)}}{2} \int_{0}^{\frac{\frac{y^{2}}{2 \sigma^{2}}}{\left(1+\frac{y^{2}}{2 \sigma^{2}}\right)}} u^{\frac{j}{2}}(1-u)^{a \alpha-\frac{j}{2}-1} d u \\
& =\frac{\left(2 \sigma^{2}\right)^{\left(1+\frac{j}{2}\right)}}{2} B \frac{\frac{y^{2}}{2 \sigma^{2}}}{\left(1+\frac{y^{2}}{2 \sigma^{2}}\right)}\left(\frac{j}{2}+1, a \alpha-\frac{j}{2}\right) .
\end{aligned}
$$

From (13), we have

$$
\Phi_{j, l}(a, b)=\frac{\left(2 \sigma^{2}\right)^{\left(1+\frac{j}{2}\right)}}{2} \int_{0}^{\infty} \frac{y^{l+1}}{\left(1+\frac{y^{2}}{2 \sigma^{2}}\right)^{\alpha b+1}} B \frac{\frac{y^{2}}{2 \sigma^{2}}}{\left(1+\frac{y^{2}}{2 \sigma^{2}}\right)}\left(\frac{j}{2}+1, a \alpha-\frac{j}{2}\right) d y
$$


where $B_{x}(p, q)=\int_{0}^{x} u^{p-1}(1-u)^{q-1} d u$. We know that

$$
B_{x}(p, q)=p^{-1} x^{p}{ }_{2} F_{1}(p, 1-q ; p+1 ; x)
$$

and

$$
\int_{0}^{1} u^{a-1}(1-u)^{b-1}{ }_{2} F_{1}(c, d ; e ; u) d u=B(a, b){ }_{3} F_{2}(c, d, a ; e, a+b ; 1)
$$

Substituting (16) and (17) in (15), we get

$$
\begin{aligned}
\Phi_{j, l}(a, b)= & \frac{\left(2 \sigma^{2}\right)\left(1+\frac{j}{2}\right)}{2} \int_{0}^{\infty} \frac{y^{l+1}}{\left(1+\frac{y^{2}}{2 \sigma^{2}}\right)^{\alpha b+1}}\left(\frac{\frac{y^{2}}{2 \sigma^{2}}}{1+\frac{y^{2}}{2 \sigma^{2}}}\right)^{\frac{j}{2}+1}\left(\frac{j}{2}+1\right)^{-1} \\
& { }_{2} F_{1}\left[\frac{j}{2}+1,1-a \alpha+\frac{j}{2}, ; \frac{j}{2}+2 ;\left(\frac{\frac{y^{2}}{2 \sigma^{2}}}{1+\frac{y^{2}}{2 \sigma^{2}}}\right)\right] d y .
\end{aligned}
$$

Setting $t=\frac{\frac{y^{2}}{2 \sigma^{2}}}{1+\frac{y^{2}}{2 \sigma^{2}}}$ in (18), we get

$$
\begin{aligned}
\Phi_{j, l}(a, b)= & \frac{\left(2 \sigma^{2}\right)^{\left(\frac{j+l}{2}+2\right)}}{2(j+2)} \int_{0}^{1} t^{\frac{j+l}{2}+1}(1-t)^{\alpha b-\frac{l}{2}-1}{ }_{2} F_{1}\left[\frac{j}{2}+1,1-a \alpha+\frac{j}{2}, ; \frac{j}{2}+2 ; t\right] d t \\
& =\frac{\left(2 \sigma^{2}\right)\left(\frac{j+l}{2}+2\right)}{2(j+2)} B\left(\frac{j+l}{2}+2, \alpha b-\frac{l}{2}\right)_{3} F_{2}\left(\frac{j}{2}+1,1-a \alpha+\frac{j}{2}, \frac{j+l}{2}+2 ; \frac{j}{2}+2, \frac{j}{2}+\alpha b+2 ; 1\right) .
\end{aligned}
$$

Lemma 2. Setting $j=0$ or $l=0$ in Lemma 1, we obtain

$$
\Phi_{0, l}(a, b)=\frac{\sigma^{2}}{a \alpha}\left[\Phi_{l}(b)-\Phi_{l}(a+b)\right]
$$

and

$$
\Phi_{j, 0}(a, b)=\frac{\sigma^{2}}{b \alpha}\left[\Phi_{j}(a+b)\right]
$$

where

$$
\Phi_{j}(a)=\int_{0}^{\infty} \frac{x^{j+1}}{\left(1+\frac{x^{2}}{2 \sigma}\right)^{a \alpha+1}} d x=\frac{\left(2 \sigma^{2}\right)^{\left(1+\frac{j}{2}\right)}}{2} B\left(a \alpha-\frac{j}{2}, 1+\frac{j}{2}\right) .
$$

Proof. Substituting $j=0$ in (13), we get

$$
\begin{aligned}
\Phi_{0, l}(a, b) & =\int_{0}^{\infty} \frac{y^{l+1}}{\left(1+\frac{y^{2}}{2 \sigma^{2}}\right)^{\alpha b+1}}\left[\int_{0}^{y} \frac{x}{\left(1+\frac{x^{2}}{2 \sigma^{2}}\right)^{a \alpha+1}} d x\right] d y \\
& =\frac{\sigma^{2}}{a \alpha} \int_{0}^{\infty} \frac{y^{l+1}}{\left(1+\frac{y^{2}}{2 \sigma^{2}}\right)^{\alpha b+1}}\left[1-\frac{1}{\left(1+\frac{y^{2}}{2 \sigma^{2}}\right)^{a \alpha}}\right] d y \\
& =\frac{\sigma^{2}}{a \alpha}\left[\Phi_{j}(b)-\Phi_{l}(a+b)\right] .
\end{aligned}
$$

Similarly, we get (20) by noting that

$$
{ }_{3} F_{2}(a, b, c ; c, d ; 1)={ }_{2} F_{1}(a, b ; d ; 1)=\frac{\Gamma(d) \Gamma(d-a-b)}{\Gamma(d-a) \Gamma(d-b)} .
$$


Theorem 1. Generalized product moments for Pareto-Rayleigh distribution are given as

$$
\mu_{r, s, n, \tilde{m}, k}^{(j, l)}=E\left[X^{j}(r, n, \tilde{m}, k) X^{l}(s, n, \tilde{m}, k)\right]=C_{s-1}\left(\frac{\alpha}{\sigma^{2}}\right)^{2}\left[\sum_{t=r+1}^{s} a_{t}^{(r)}(s)\left(\sum_{i=1}^{r} a_{i}(r) \Phi_{j, l}\left(\gamma_{i}-\gamma_{t}, \gamma_{t}\right)\right)\right] .
$$

Proof. We have

$$
\mu_{r, s, n, \tilde{m}, k}^{(j, l)}=C_{s-1} \int_{0}^{\infty} \int_{0}^{y} x^{j} y^{l}\left[\sum_{i=r+1}^{s} a_{i}^{(r)}(s)\left\{\frac{\bar{F}(y)}{\bar{F}(x)}\right\}^{\gamma_{i}}\right]\left(\sum_{i=1}^{r} a_{i}(r)\{\bar{F}(x)\}^{\gamma_{i}}\right) \frac{f(x)}{\bar{F}(x)} \frac{f(y)}{\bar{F}(y)} d x d y .
$$

which yields (21).

Corollary 2. Product moment for Pareto-Rayleigh distribution, when $m_{1}=m_{2}=\cdots=m_{n-1}=m \neq-1$ is given as

$$
\begin{aligned}
\mu_{r, s, n, m, k}^{(j, l)} & =E\left[X^{j}(r, n, m, k) X^{l}(s, n, m, k)\right] \\
& =\frac{C_{s-1}}{(r-1) !(s-r-1) !(m+1)^{s-2}}\left(\frac{\alpha}{\sigma^{2}}\right)^{2} \sum_{i=0}^{r-1} \sum_{t=0}^{s-r-1}(-1)^{i+t}\left(\begin{array}{c}
r-1 \\
i
\end{array}\right)\left(\begin{array}{c}
s-r-1 \\
t
\end{array}\right) \Phi_{j, l}\left(\gamma_{r-i}-\gamma_{s-t}, \gamma_{s-t}\right) .
\end{aligned}
$$

Remark 1. Setting $m_{1}=m_{2}=\cdots=m_{n-1}=0$ and $k=1$ in (22), we get the result as the product moment of order statistics as

$$
\begin{aligned}
\mu_{r, s, n, 0,1}^{(j, l)} & =\mu_{r, s: n}^{j, l} \\
& =\frac{C_{s-1}}{(r-1) !(s-r-1) !}\left(\frac{\alpha}{\sigma^{2}}\right)^{2} \sum_{i=0}^{r-1} \sum_{t=0}^{s-r-1}(-1)^{i+t}\left(\begin{array}{c}
r-1 \\
i
\end{array}\right)\left(\begin{array}{c}
s-r-1 \\
t
\end{array}\right) \Phi_{j, l}(s-r-t+i, n-s+t+1) .
\end{aligned}
$$

Corollary 3. Single moments of the Pareto-Rayleigh distribution are of the form

$$
\mu_{s, n, \tilde{m}, k}^{(l)}=C_{s-1}\left(\frac{\alpha}{\sigma^{2}}\right) \sum_{i=1}^{s} a_{i}(s) \Phi_{l}\left(\gamma_{i}\right) .
$$

Proof. Putting $j=0$ in (21) and using (19), we get

$$
\begin{aligned}
\mu_{r, s, n, \tilde{m}, k}^{(l)}= & C_{s-1}\left(\frac{\alpha}{\sigma^{2}}\right)\left[\sum_{t=r+1}^{s} \frac{a_{t}^{(r)}(s)}{\left(\gamma_{i}-\gamma_{t}\right)}\left(\sum_{i=1}^{r} a_{i}(r)\left\{\Phi_{l}\left(\gamma_{t}\right)-\Phi_{l}\left(\gamma_{i}\right)\right\}\right)\right] . \\
\mu_{s, n, \tilde{m}, k}^{(l)}= & C_{s-1}\left(\frac{\alpha}{\sigma^{2}}\right)\left[\sum_{t=r+1}^{s} a_{t}^{(r)}(s) \Phi_{l}\left(\gamma_{t}\right)\left(\sum_{i=1}^{r} \frac{a_{i}(r)}{\left(\gamma_{i}-\gamma_{t}\right)}\right)\right] \\
& +C_{s-1}\left(\frac{\alpha}{\sigma^{2}}\right)\left[\sum_{i=1}^{r} a_{i}(r) \Phi_{l}\left(\gamma_{i}\right)\left(\sum_{t=r+1}^{s} \frac{a_{t}^{(r)}(s)}{\left(\gamma_{i}-\gamma_{t}\right)}\right)\right] .
\end{aligned}
$$

Now using the results found in [20] we obtain

$$
\sum_{i=1}^{r} \frac{a_{i}(r)}{\left(\gamma_{i}-\gamma_{j}\right)}=\prod_{j=1}^{r} \frac{1}{\left(\gamma_{i}-\gamma_{j}\right)}, \quad j \neq i, \quad \gamma_{j} \neq \gamma_{i}, \quad 1 \leq i \leq r \leq n,
$$

and

$$
\sum_{i=r+1}^{s} \frac{a_{i}^{(r)}(s)}{\left(\gamma_{i}-\gamma_{j}\right)}=\prod_{j=r+1}^{s} \frac{1}{\left(\gamma_{i}-\gamma_{j}\right)}, \quad j \neq i, \quad \gamma_{j} \neq \gamma_{i}, \quad r+1 \leq i \leq s \leq n .
$$

Hence,

$$
\mu_{s, n, \tilde{m}, k}^{(l)}=C_{s-1}\left(\frac{\alpha}{\sigma^{2}}\right)\left[\sum_{t=r+1}^{s} a_{t}^{(r)}(s) \Phi_{l}\left(\gamma_{t}\right)\left(\prod_{j=1}^{r} \frac{1}{\left(\gamma_{i}-\gamma_{j}\right)}\right)\right]
$$




$$
+C_{s-1}\left(\frac{\alpha}{\sigma^{2}}\right)\left[\sum_{i=1}^{r} a_{i}(r) \Phi_{l}\left(\gamma_{i}\right)\left(\prod_{j=r+1}^{s} \frac{1}{\left(\gamma_{i}-\gamma_{j}\right)}\right)\right]
$$

which yields (24).

Corollary 4. Corollary2.3 Single moments of gos for Pareto-Rayleigh distribution, when $m_{1}=m_{2}=\cdots=m_{n-1}=$ $m \neq-1$, are given as

$$
\mu_{s, n, m, k}^{(l)}=\frac{C_{s-1}}{(s-1) !} \frac{1}{(m+1)^{s-1}}\left(\frac{\alpha}{\sigma^{2}}\right) \sum_{i=0}^{s-1}(-1)^{i}\left(\begin{array}{c}
r-1 \\
i
\end{array}\right) \Phi_{j}\left(\gamma_{s-i}\right) .
$$

Proof. Setting $m_{1}=m_{2}=\cdots=m_{n-1}=m \neq-1$ in (24) and using (7) we get the result as the single moment.

Remark 2. Setting $m_{1}=m_{2}=\cdots=m_{n-1}=0$ and $k=1$ in (25), we get the result as the single moment from order statistics

$$
\mu_{s, n, 0,1}^{(l)}=\mu_{s: n}^{(l)}=\frac{C_{s-1}}{(s-1) !}\left(\frac{\alpha}{\sigma^{2}}\right) \sum_{i=0}^{s-1}(-1)^{i}\left(\begin{array}{c}
s-1 \\
i
\end{array}\right) \Phi_{j}(n-s+i+1) .
$$

Remark 3. Setting $j=0$ and $l=0$ in $(21)$ we get

$$
\sum_{i=1}^{r} \sum_{t=r+1}^{s} \frac{a_{i}(r) a_{t}^{r}(s)}{\gamma_{i} \gamma_{t}}=\frac{1}{C_{s-1}}
$$

and setting $l=0$ in (24) we obtain

$$
\sum_{i=1}^{r} \frac{a_{i}(r)}{\gamma_{i}}=\frac{1}{C_{r-1}}
$$

Combining (27) and (28), we get another identity,

$$
\sum_{t=r+1}^{s} \frac{a_{t}^{r}(s)}{\gamma_{t}}=\frac{C_{r-1}}{C_{s-1}}
$$

When $m_{1}=m_{2}=\cdots=m_{n-1}=m \neq-1$, (29) reduces to another identity

$$
\sum_{t=0}^{s-r-1}(-1)^{t}\left(\begin{array}{c}
s-r-1 \\
t
\end{array}\right) \frac{1}{\gamma_{s-t}}=\frac{C_{r-1}(s-r-1) !(m+1)^{s-r-1}}{C_{s-1}}
$$

which is obtained in [3].

Remark 4. Setting $\gamma_{r}=k+n-r+\sum_{i=r}^{l} m_{j}, 1 \leq r \leq l \leq n, m_{i} \in N$, in (21), then the product moments of progressive type II censored order statistics of Pareto-Rayleigh distribution can be obtained.

\section{Numerical Computations}

Here we have calculated means and variances for order statistics (Table $1 \& 2$ ), and generalized order statistics (gos) (Table 3 \& 4). All computations here we obtained using Mathematica. Mathematica like other algebraic manipulation packages allow for arbitrary precisions, so the accuracy of the given values is not an issue. In case of order statistics, the relation $\sum_{r=1}^{n} \mu_{r, n, 0,1}^{j}=n \mu_{1,1,0,1}^{j}, \quad j=1,2$, is used to evaluate the means and variancess, see [21]. It is observed that when the sample size $n$ is fixes, increasing the value of $r$ directly increases the means and variances, whereas, for fixed $r$, the opposite occurs in the case when the sample size $n$ increases. 
Table 1. Means of order statistics from Pareto-Rayleigh distribution $(\alpha=2, \sigma=1)$

\begin{tabular}{l|l|l|l|l|l|l|l|l}
\multicolumn{7}{|c|}{} & \multicolumn{7}{c}{$\mathrm{n}$} \\
\hline $\mathrm{r}$ & 1 & 2 & 3 & 4 & 5 & 6 & 7 & 8 \\
\hline 1 & 1.1107 & 0.6942 & 0.5469 & 0.4653 & 0.4120 & 0.3736 & 0.3443 & 0.3209 \\
\hline 2 & & 1.5272 & 0.9892 & 0.7907 & 0.6786 & 0.6040 & 0.5496 & 0.5078 \\
\hline 3 & & & 1.7962 & 1.1877 & 0.9589 & 0.8279 & 0.7398 & 0.6752 \\
\hline 4 & & & & 1.9991 & 1.3403 & 1.0900 & 0.9453 & 0.8474 \\
\hline 5 & & & & & 2.1638 & 1.4654 & 1.1984 & 1.0433 \\
\hline 6 & & & & & & 2.3035 & 1.5722 & 1.2916 \\
\hline 7 & & & & & & & 2.4253 & 1.6658 \\
\hline 8 & & & & & & & & 2.5339
\end{tabular}

Table 2. Variances of order statistics from Pareto-Rayleigh distribution $(\alpha=2, \sigma=1)$

\begin{tabular}{l|l|l|l|l|l|l|l|l} 
& \multicolumn{7}{c}{$\mathrm{n}$} \\
\hline $\mathrm{r}$ & 1 & 2 & 3 & 4 & 5 & 6 & 7 & 8 \\
\hline 1 & 0.7663 & 0.1847 & 0.1011 & 0.0692 & 0.0525 & 0.0422 & 0.0353 & 0.0303 \\
\hline 2 & & 1.0009 & 0.2214 & 0.1176 & 0.0792 & 0.0594 & 0.0475 & 0.0395 \\
\hline 3 & & & 1.1735 & 0.2464 & 0.1281 & 0.0852 & 0.0635 & 0.0504 \\
\hline 4 & & & & 1.3180 & 0.2672 & 0.1366 & 0.0900 & 0.0666 \\
\hline 5 & & & & & 1.4450 & 0.2855 & 0.1441 & 0.0942 \\
\hline 6 & & & & & & 1.5599 & 0.3021 & 0.1509 \\
\hline 7 & & & & & & & 1.6655 & 0.3175 \\
\hline 8 & & & & & & & & 1.7639
\end{tabular}

Table 3. Means of gos from Pareto-Rayleigh distribution $(\alpha=2, \sigma=1, m=1, k=2)$

\begin{tabular}{l|l|l|l|l|l|l|l|l}
\multicolumn{7}{l|}{} & \multicolumn{7}{c}{$\mathrm{n}$} \\
\hline $\mathrm{r}$ & 1 & 2 & 3 & 4 & 5 & 6 & 7 & 8 \\
\hline 1 & 0.3471 & 0.2327 & 0.1868 & 0.1605 & 0.1428 & 0.1300 & 0.1200 & 0.1121 \\
\hline 2 & & 0.2308 & 0.1622 & 0.1329 & 0.1155 & 0.1036 & 0.0947 & 0.0878 \\
\hline 3 & & & 0.1325 & 0.0957 & 0.0795 & 0.0697 & 0.0628 & 0.0577 \\
\hline 4 & & & & 0.0724 & 0.0532 & 0.0447 & 0.0394 & 0.0357 \\
\hline 5 & & & & & 0.0386 & 0.0288 & 0.0243 & 0.0216 \\
\hline 6 & & & & & & 0.0203 & 0.0153 & 0.0130 \\
\hline 7 & & & & & & & 0.0105 & 0.0080 \\
\hline 8 & & & & & & & & 0.0054
\end{tabular}

Table 4. Variances of gos from Pareto-Rayleigh distribution $(\alpha=2, \sigma=1, m=1, k=2)$

\begin{tabular}{l|l|l|l|l|l|l|l|l}
\multicolumn{7}{|c|}{} & \multicolumn{7}{c}{$\mathrm{n}$} \\
\hline $\mathrm{r}$ & 1 & 2 & 3 & 4 & 5 & 6 & 7 & 8 \\
\hline 1 & 0.2128 & 0.0887 & 0.0560 & 0.0409 & 0.0322 & 0.0266 & 0.0226 & 0.0197 \\
\hline 2 & & 0.2086 & 0.0971 & 0.0641 & 0.0481 & 0.0385 & 0.0321 & 0.0275 \\
\hline 3 & & & 0.1480 & 0.0733 & 0.0499 & 0.0380 & 0.0308 & 0.0259 \\
\hline 4 & & & & 0.0914 & 0.0471 & 0.0327 & 0.0253 & 0.0207 \\
\hline 5 & & & & & 0.0527 & 0.0280 & 0.0197 & 0.0154 \\
\hline 6 & & & & & & 0.0292 & 0.0158 & 0.0113 \\
\hline 7 & & & & & & & 0.0158 & 0.0087 \\
\hline 8 & & & & & & & & 0.0084
\end{tabular}

Author Contributions: All authors contributed equally to the writing of this paper. All authors read and approved the final manuscript.

Conflicts of Interest: "The authors declare no conflict of interest."

\section{References}


[1] Kamps, U. (1995). A Concept of Generalized Order Statistics. B. G. Teubner Stuttgart, Germany.

[2] Kamps, U., \& Cramer, E. (2001). On distributions of generalized order statistics. Statistics, 35, 269-280.

[3] Khan, A. H., \& Khan, M. J. S. (2012). On ratio and inverse moment of generalized order statistics from Burr distribution. Pakistan Journal of Statistics, 28, 59-68.

[4] Alzaatreh, A., Lee, C., \& Famoye, F. (2013). A new method for generating families of continuous distributions. Metron, 71(1), 63-79.

[5] Jebeli, M., \& Deiri, E. (2020). Estimation methods for the probability density function and the cumulative distribution function of the Pareto-Rayleigh distribution. Statistics, 54(1), 135-151.

[6] Al-Zahrani, B., \& Al-Sobhi, M. (2013). On parameters estimation of Lomax distribution under general progressive censoring. Journal of Quality and Reliability Engineering, 2013, Article ID 431541. https:/ / doi.org/10.1155/2013/431541.

[7] Rao, R. S., Kantam, R. R. L., \& Prasad, G. (2015). Modified maximum likelihood estimation in pareto-rayleigh distribution. Golden Research Thoughts, 140-152.

[8] Rao, R. S. (2016). Discriminating between size biased Lomax distribution and Pareto-Rayleigh distribution. Open Journal of Applied and Theoretical Mathematics, 2, 409-418.

[9] Parsad, G., Rao, R. S., \& Kantam, R. R. I. (2016). Acceptance sampling based on life tests: Pareto-Rayleigh model. International Journal of Advanced Research in Science, Engineering and Technology, 5, 427-436.

[10] Athar, H., \& Nayabuddin. (2014). A note on exact moments of order statistics from exponentiated log-logistic distribution. ProbStat Forum, 7, 39-44.

[11] Khan, M. J. S., Sharma, A., Khan, M. I., \& Kumar, S. (2015). Exact moments of record values from Burr distribution with applications. International Journal of Computational and Theoretical Statistics, 2, 107-115.

[12] Ahmad, A. E. B. A. (2008). Single and product moments of generalized order statistics from linear exponential distribution. Communications in Statistics-Theory and Methods, 37(8), 1162-1172.

[13] Khan, R. U., Kumar, D., \& Athar, H. (2010). Moments of generalized order statistics from Erlang-Truncated exponential distribution and its charactersics. International Journal of Statistics and Systems, 5, 455-464.

[14] Kumar, D., \& Khan, R. U. (2014). Moments of power function distribution based on ordered random variables and characterization. Sri Lankan Journal of Applied Statistics, 15, 91-105.

[15] Kumar, D. (2015). Exact moments of generalized order statistics from type II exponentiated loglogistic distribution. Hacettepe Journal of Mathematics and Statistics, 44, 715-733.

[16] Kumar, D., \& Dey, S. (2017). Relations for moments of generalized order statistics from extended exponential distribution. American Journal of Mathematical and Management Sciences, 36, 378â€“400.

[17] Malik, M.R. and Kumar, D. (2019): Generalized Pareto distribution based on generalized order statistics and associated inference, Statistics in Transition new Series, 20, 57-79.

[18] Singh, B., Khan, R. U., \& Khan, M. A. (2019). Characterizations of q-Weibull distribution based on generalized order statistics Journal of Statistics and Management Systems, 22, 1573-1595.

[19] Kumar, D., Nassar, M., \& Dey, S. (2020). Inference for generalized inverse Lindley distribution based on generalized order statistics, Afrika Matematika, 31, 1207-1235.

[20] Bieniek, M., \& Szynal, D. (2003). Characterizations of distributions via linearity of regression of generalized order statistics. Metrika, 58(3), 259-271.

[21] Joshi, P. C., \& Balakrishnan, N. (1982). Recurrence relations and identities for the product moments of order statistics. Sankhya: The Indian Journal of Statistics, Series B, 44 39-49. 